Enferm Bras 2020;19(3):211-9

https://doi.org/10.33233/eb.v19i3.3072

\title{
ARTIGO ORIGINAL \\ Incidência de infecção de sítio cirúrgico em um hospital do interior de Rondônia
}

Pâmella Polastry Braga Amaral ${ }^{*}$, Aline de Souza Coelho**, Thaís Araújo da Silva***, Daiany Cristina Gil Glioli Custódio****, Orlete Donato de Oliveira Miranda ${ }^{* \star \star *}$

${ }^{*}$ Enfermeira, Centro Universitário São Lucas Educacional, ${ }^{* *}$ Enfermeira, Centro Universitário São Lucas Educacional, Residente em Saúde da Família e da Comunidade, ${ }^{* * *}$ Enfermeira, Faculdade Panamericana de Ji-Paraná, ${ }^{* * * *}$ Enfermeira, Centro Universitário São Lucas Educacional, Residente em Urgência e Emergência, ${ }^{* * * *}$ Enfermeira, Centro Universitário Luterano de JiParaná, Mestranda em Promoção da Saúde Desenvolvimento Humano e Sociedade / ULBRA/RS Especialista em Gestão Hospitalar, Universidade Paulista, Especialista em Qualidade e Segurança no cuidado ao Paciente, Instituto Sírio Libanês

Recebido em 8 de julho de 2019; aceito em 16 de junho de 2020.

Correspondência: Aline de Souza Coelho, Afonso Juca de Oliveira, 4689, Jardim Eldorado, 76987-092 Vilhena RO

Aline de Souza Coelho: alinecoelho1994@gmail.com

Pâmella Polastry Braga Amaral: pamellapolastry@gmail.com

Daiany Cristina Gil Glioli Custódio: daianycgcustodio@gmail.com

Thaís Araújo: siatharaujo@gmail.com

Orlete Donato de Oliveira Miranda: orletedonato@gmail.com

\section{Resumo}

As Infecções de Sítio Cirúrgico caracterizam-se como complicações decorrentes de procedimentos que se apresentam sempre no período pós-operatório mediato, podendo acometer tecidos, órgão ou cavidades. O objetivo do presente estudo foi determinar a incidência de infecção do sítio cirúrgico em um hospital municipal do interior de Rondônia. Trata-se de um estudo quantitativo, transversal, exploratório e descritivo, realizado com 106 pacientes submetidos a procedimentos cirúrgicos eletivos, no período de agosto de 2017 a março de 2018, em uma instituição pública do interior de Rondônia, por meio da aplicação de questionários, levantamento de dados de prontuário e monitorização durante o oitavo e trigésimo dia de pósoperatório, por meio de contato telefônico. A incidência de infecção na amostra foi 7,5\%; sendo os fatores de risco, etilismo, doenças crônicas e tabagismo; todos apresentaram correlação positiva fraca à correlação de Pearson, com exceção da escolaridade que apresentou correlação negativa fraca. A taxa de incidência determinada por este estudo encontra-se acima do recomendado pelas autoridades sanitárias, fazendo-se urgente a promoção de medidas objetivando melhorar o controle de infecção e a segurança do paciente.

Palavras-chave: procedimento cirúrgico, fatores de risco, infecção, incidência.

\section{Abstract \\ Incidence of surgical site infection in a hospital in the interior of Rondônia}

Surgical Site Infections are characterized as complications arising from procedures that always present in the mid postoperative period, and can affect tissues, organs or cavities. The objective of the present study was to determine the incidence of surgical site infection in a municipal hospital in the interior of Rondônia. This is a quantitative, transversal, exploratory and descriptive study carried out with 106 patients submitted to elective surgical procedures, between August 2017 and March 2018, at a public institution in the interior of Rondônia, using questionnaires, survey data collection and monitoring during the eighth and thirtieth postoperative day, through telephone contact. The incidence of infection in the sample was $7.5 \%$; being the risk factors, alcoholism, chronic diseases and smoking; all showed a weak positive correlation, with Pearson's correlation, except for the schooling that presented weak negative correlation. The incidence rate determined by this study is above the recommendation by health authorities, and it is urgent to promote measures aimed at improving infection control and patient safety. 
Keywords: surgical procedure, risk factors, infection, incidence.

\section{Resumen \\ Incidencia de infección quirúrgica en un hospital en el interior de Rondônia}

Las infecciones del sitio quirúrgico se caracterizan como complicaciones resultantes de procedimientos que siempre están presentes en el período postoperatorio mediato y pueden afectar tejidos, órganos o cavidades. El objetivo del presente estudio fue determinar la incidencia de infección del sitio quirúrgico en un hospital municipal en el interior de Rondônia. Este es un estudio cuantitativo, transversal, exploratorio y descriptivo, realizado con 106 pacientes sometidos a procedimientos quirúrgicos electivos, desde agosto de 2017 hasta marzo de 2018, en una institución pública en el interior de Rondônia, mediante la aplicación de cuestionarios, recopilación de datos de registros médicos y monitoreo durante el octavo y trigésimo día postoperatorio, por contacto telefónico. La incidencia de infección en la muestra fue del 7,5\%; siendo los factores de riesgo, alcoholismo, enfermedades crónicas y tabaquismo; todos mostraron una correlación positiva débil, a la correlación de Pearson, a excepción de la educación, que presentó una correlación negativa débil. La tasa de incidencia determinada por este estudio es superior a la recomendada por las autoridades sanitarias, por lo que es urgente promover medidas destinadas a mejorar el control de infecciones y la seguridad del paciente.

Palabras-clave: procedimiento quirúrgico, factores de riesgo, infección, incidencia.

Introdução

O desenvolvimento de infecções relacionadas à assistência à saúde (IRAS), constantemente é abordado como causa de morbimortalidade, e causam inúmeros impactos relacionados à evolução dos pacientes submetidos a algum procedimento cirúrgico, independentemente do objetivo da abordagem, podendo ser curativa, diagnóstica ou preventiva [1].

As Infecções de Sítio Cirúrgico (ISC) caracterizam-se como complicações decorrentes de procedimentos que se apresentam sempre no período pós-operatório mediato, podendo acometer tecidos, órgãos ou cavidades que foram abordados durante o procedimento. No Brasil, permanecem dentre as infecções mais prevalentes em meio às IRAS [2].

Existem determinados fatores que são identificados como de risco para o seu desencadeamento, dentre estes fatores estão os intrínsecos relacionados ao paciente, e extrínsecos que compreendem fatores relacionados ao procedimento cirúrgico e a abordagem realizada [3].

Dentre todos os procedimentos cirúrgicos que ocorrem no Brasil, cerca de $11 \%$ das cirurgias realizadas são afetadas pela ISC. Este número pode variar na presença de determinados fatores como o tipo de procedimento e a condição imune do paciente. Frequentemente, essas são apontadas como a causa principal de complicações pós-operatórias [4].

A enfermagem contribui no controle destas, por meio de profissionais dotados de olhar crítico, promovendo ações de controle para diminuição de sua propagação. Através da educação continuada, os enfermeiros realizam ações que visam repassar orientações e informações que veem a contribuir com a diminuição de tal incidência [5].

O número de cirurgias eletivas no Brasil vem aumentando gradualmente, sendo representado por um crescimento de $11,7 \%$ no período de dois anos, passando de 2.120 .580 procedimentos realizados em 2012 para 2.370.039 no ano de 2014. Em 2015 o Ministério da Saúde disponibilizou cerca de $R$ \$ 143,2 milhões aos estados e municípios para o emprego na realização de procedimentos cirúrgicos eletivos no Sistema Único de Saúde (SUS). Para o estado de Rondônia, o valor encaminhado foi de $\mathrm{R} \$ 626.991,89$ [6]. Em 2017, o valor liberado saltou para 250 milhões distribuídos entre estados e munícipios [7].

Uma maior demanda de procedimentos cirúrgicos sugere um aumento de possíveis pacientes com complicações relacionados aos mesmos, dentre elas a ISC, que ocorre frequentemente nas unidades hospitalares. Segundo a ANVISA, de todas as infecções relacionadas à assistência à saúde (IRAS), $14 \%$ a $16 \%$ do total trata-se de ISC, ocupando a terceira posição em relação a todas as outras infecções que afetam os serviços de saúde [8].

A identificação da incidência de infeção e dos fatores de risco envolvidos no surgimento de infecções de sítio cirúrgico possibilita cooperar com a diminuição de casos de ISC, pois por meio das modificações nas ações tanto dos profissionais como dos pacientes, por meio de ações de educação em saúde, pode resultar na diminuição da incidência destes casos. 
O objetivo geral do presente estudo foi determinar a incidência de infecção do sítio cirúrgico em um hospital municipal do interior de Rondônia.

\section{Material e métodos}

Trata-se de um estudo quantitativo, transversal, exploratório e descritivo de 106 pacientes submetidos a procedimentos cirúrgicos eletivos, realizados durante o período de agosto de 2017 a março de 2018, no hospital municipal de Ji-Paraná/RO. Os critérios de inclusão foram pacientes submetidos à procedimentos cirúrgicos eletivos, ambos os sexos, que aceitaram disponibilizar contato telefônico. Os critérios de exclusão foram pacientes submetidos a procedimentos de urgência e emergência, ou que não possuíam condições de autorizar a pesquisa, devido ao efeito de drogas, que impossibilitassem respostas coerentes à entrevista, procedimentos cirúrgicos classificados como infectados, reabordagem cirúrgica e pacientes sob efeito de drogas,

O instrumento utilizado para coleta de dados constituiu em um questionário adaptado do artigo do autor Aguiar et al. [3]: "Fatores associados à infecção de sítio cirúrgico em um hospital na Amazônia ocidental brasileira". O questionário foi composto de investigação de dados sociodemográficos, e questões relacionadas aos momentos pré-operatório, intraoperatório e pós-operatório.

Além do questionário, foi realizada coleta de dados do prontuário para complementar as respostas dos pacientes e de seus acompanhantes. Após esta etapa, já na alta do paciente, foi estabelecido contato telefônico, no oitavo e trigésimo dia de pós-operatório, e os pesquisados foram questionados a respeito de retornos hospitalares e presença de sinais flogísticos.

O contato realizado visava investigar a presença de alterações na ferida operatória (FO), descrevendo suas características, e a necessidade de retorno hospitalar, perante alguma alteração na mesma.

O presente estudo cumpriu os preceitos éticos legais da pesquisa em seres humanos, sendo submetido à previa apreciação ética conforme resolução 466/12 do Ministério da Saúde. O trabalho foi aprovado através do parecer 2.195.12. Os pesquisados foram devidamente esclarecidos em relação aos objetivos da pesquisa e manifestaram seu consentimento por meio da assinatura do Termo de Consentimento Livre e Esclarecido (TCLE).

Os dados coletados foram tabulados e calculados seguindo a orientação do cálculo de incidência da ANVISA, utilizado para determinar a taxa de incidência, analisados por método de estatística descritiva simples e realizado correlação de Pearson.

Resultados

Este estudo identificou uma incidência de ISC de 7,5\% na amostra, sendo 5,3\% entre os pesquisados do sexo feminino e de $2,1 \%$ entre os pesquisados do sexo masculino. O perfil dos pacientes está disposto na Tabela I.

Tabela I - Perfil socioeconômico dos pacientes selecionados.

\begin{tabular}{llcc}
\hline Variáveis & Perfil & № & $\%$ \\
\hline Sexo & Feminino & 76 & $72 \%$ \\
& Masculino & 30 & $28 \%$ \\
\hline Idade & & & $4 \%$ \\
& $0-17$ & 4 & $39 \%$ \\
& $18-39$ & 41 & $41 \%$ \\
\hline Estado civil & $40-59$ & 43 & $17 \%$ \\
& 60 ou mais & 18 & $22 \%$ \\
& Solteiro & 23 & $53 \%$ \\
& Casado & 56 & $10 \%$ \\
& União estável & 11 & $9 \%$ \\
& Divorciado & 10 & $6 \%$ \\
\hline Escolaridade & Viúvo & 6 & $8 \%$ \\
& Superior completo & 9 & $1 \%$ \\
& Superior incompleto & 1 & $4 \%$ \\
& Téc. Completo & 4 & $23 \%$
\end{tabular}




\begin{tabular}{llcc} 
& Fund. Completo & 18 & $17 \%$ \\
& Fund. Incompleto & 34 & $32 \%$ \\
& Não frequentou escola & 8 & $7 \%$ \\
\hline Cor & Pardo & 62 & $58 \%$ \\
& Negro & 12 & $11 \%$ \\
& Branco & 25 & $24 \%$ \\
& Amarelo & 7 & $7 \%$ \\
\hline Moradia & Alvenaria & 64 & $60 \%$ \\
& Madeira & 35 & $33 \%$ \\
& Mista & 7 & $7 \%$ \\
\hline
\end{tabular}

Fonte: Própria

Além de um breve perfil, a pesquisa também buscou elencar outros fatores intrínsecos que podem estar relacionados ao surgimento de ISC, para isso foram abordados tais fatores dispostos na tabela II.

Tabela II - Presença de fatores intrínsecos.

\begin{tabular}{lcccc}
\hline Fatores & Sim $\left(\mathbf{n}^{\circ}\right)$ & Não $\left(\mathbf{n} \mathbf{o}^{-}\right.$ & Sim (\%) & Não (\%) \\
\hline Etilista & 20 & 85 & $19 \%$ & $81 \%$ \\
\hline Tabagismo & 10 & 96 & $9 \%$ & $91 \%$ \\
\hline Doenças crônicas & 34 & 72 & $32 \%$ & $68 \%$ \\
\hline Outros procedimentos & 77 & 29 & $73 \%$ & $27 \%$ \\
\hline Fonte: Própria & & & &
\end{tabular}

Os pacientes que relataram alguma doença crônica representam $34 \%$, e a doença mais relatada foi a hipertensão arterial (25\%), seguida de doença renal $(7 \%)$ e diabetes mellitus $(5 \%)$, fibromialgia (1\%), anemia (1\%), alergia crônica (1\%), cefaleia crônica (1\%), hipercolesterolemia $(1 \%)$, sinusite (1\%), Alzheimer (1\%).

Em relação aos procedimentos pré-operatórios, $86 \%$ dos pesquisados realizaram tricotomia, $99 \%$ tomaram banho e 100\% dos pacientes permaneceram em jejum pelo menos 8 horas antes do procedimento.

Referente à internação pré-operatória, $95 \%$ dos pacientes foram internados no mesmo dia que foram submetidos ao procedimento, os outros $5 \%$ permaneceram internados até dois dias antes da cirurgia.

Todos procedimentos cirúrgicos realizados e selecionados durante o período da pesquisa, estão distribuídos na tabela III.

Tabela III - Classificação dos procedimentos cirúrgicos selecionados para pesquisa.

\begin{tabular}{lcc}
\hline Classificação & № & $\%$ \\
\hline Finalidade cirúrgica & Paliativa $=90$ & $85 \%$ \\
& Radical=14 & $13 \%$ \\
& Diagnóstica=2 & $2 \%$ \\
\hline Risco cardiológico & Pequeno=10 & $9 \%$ \\
& Médio=92 & $87 \%$ \\
\hline Duração da cirurgia & Grande=4 & $4 \%$ \\
\hline Potencial de contaminação & Porte I=101 & $95 \%$ \\
& Porte II= 5 & $5 \%$ \\
\hline & Limpa $=36$ & $34 \%$ \\
& Potencialmente Cont.=64 & $60 \%$ \\
\hline
\end{tabular}

Fonte: Própria

Referente à classificação da American Society Anestesiologist (ASA), foram classificados como ASA I (71\%), ASA II (26\%) e ASA III apenas 3\%.

No pós-operatório $22 \%$ dos pesquisados fizeram uso de sonda vesical de demora (SVD) e 15\% precisaram usar drenos tubulares ou laminares. Antibioticoprofilaxia no pós-operatório foi detectado em $98 \%$ das prescrições.

O contato telefônico com os pacientes no oitavo e trigésimo dia revelaram que $16 \%$ alegaram ter retornado para realizar a retirada dos pontos, $14 \%$ afirmaram ter retornado devido a alguma intercorrência. Quando questionados em relação aos sinais flogísticos, $17 \%$ referiram algum sinal. Já no trigésimo dia, quando restabelecido contato, 9\% ainda relataram queixas 
relacionadas ao procedimento cirúrgico, sendo nesses clientes no retorno hospitalar identificado infecção cirúrgica em $7,5 \%$ dos casos.

Os dados levantados foram analisados estatisticamente através da correlação de Person. Os fatores correlacionados neste método foram dispostos na tabela IV.

Tabela IV - Variáveis aplicadas conforme a correlação de Pearson.

\begin{tabular}{lcc}
\hline Fatores correlacionados a infecção & Frequência (\%)r & $\mathbf{r}^{\mathbf{2}}$ \\
\hline Doenças crônicas & $32 \%$ & 0,26 \\
Hipertensão arterial sistêmica & $25 \%$ & 0,16 \\
Retorno médico & $30 \%$ & 0,15 \\
Etilismo & $19 \%$ & 0,13 \\
Tabagismo & $13 \%$ & 0,02 \\
Baixa escolaridade & $57 \%$ & $-0,2$ \\
\hline
\end{tabular}

Fonte: Dados da pesquisa, 2018.

Discussão

A incidência de infecção foi de $7,5 \%$, resultado inferior à média nacional (11\%) [4] e superior a outro estudo realizado na região $(3,6 \%)$ [3]. Observa-se ainda que a procura feminina foi superior à masculina, justificada em estudos os quais revelam que homens procuram com menor frequência atendimento, deste modo, é mais frequente a presença de mulheres nos locais designados ao cuidado da saúde do que homens [9].

Em relação às idades acometidas pela ISC, a faixa etária mais acometida 40-59 (63\%), sendo o valor mais significativo. Em estudo anterior aponta o processo de senescência como fator que contribui com aumento de chances de complicações relacionadas ao ato cirúrgico, principalmente os pacientes com idade superior a 60 anos. São as mudanças fisiológicas caraterísticas deste processo que contribui com um risco mais elevado [10].

As idades extremas não foram as mais acometidas, porém a maior faixa etária afetada foi composta por pessoas que estão aproximando-se da terceira idade, sugerindo assim que o processo de senescência contribui com o surgimento de tal.

Seguindo com os dados demográficos também foram analisadas as condições de moradia e escolaridade dos indivíduos. Dentro do grupo que apresentou ISC não houve pacientes com escolaridade maior que o ensino fundamental completo, a maioria (50\%) não completou o ensino fundamental. Os dados relatados condizem com a literatura, uma vez que ela aponta que a baixa escolaridade, assim como níveis de condições socioeconômicas não favoráveis tornam o paciente mais suscetível [3].

A amostra atingida pela ISC se encaixa neste perfil de baixa escolaridade, sendo sugerível que estes tenham maior suscetibilidade a ISC. Quando analisado estatisticamente a variável escolaridade, encontrou-se uma correlação inversa muito fraca, que significa quanto mais baixa a escolaridade dos indivíduos, maior o risco de desenvolver ISC, porém, como foi uma correlação muito fraca, este elemento não é determinante para o seu desencadeamento.

Quanto aos maus hábitos de vida dentro do grupo que apresentou ISC, 38\% dos pacientes eram etilistas e 13\% eram tabagistas. Estudo anterior realizado em um hospital geral em Fortaleza, ao analisar estas variáveis, identificaram associações significativas, sendo representada em percentuais elevados dentro do grupo que desenvolveu ISC, chegando a $69 \%$ em relação ao tabagismo e $35 \%$ relacionado ao etilismo [11].

Desta forma, estes fatores não apresentaram números tão significativos como na pesquisa anterior, este acontecimento pode ser atribuído aos poucos pacientes que referiram tais hábitos. Quando analisado estatisticamente, o tabagismo não possuiu valor significativo, não estando relacionado ao surgimento de ISC.

E quanto ao etilismo aplicado à correlação de Pearson, o resultado mostra uma correlação muito fraca, ou seja, este hábito por si só não é capaz de resultar num processo de ISC, porém, se relacionado a outros fatores pode ter alguma influência.

O tabaco aumenta o risco de infecção do sítio cirúrgico, retarda o processo de cicatrização e ainda tem a capacidade de aumentar as deiscências de sutura. Sendo essenciais orientações em relação à suspensão do fumo no período pré-operatório [12].

No estudo dos que adquiriram infeções do sítio cirúrgico, $75 \%$ eram portadores de doenças crônicas. A presença de doenças crônicas é apontada como um fator de risco para o desencadeamento de ISC. Estudos recentes apontam a relação de doenças sistêmicas com o surgimento de ISC no pós-operatório [13]. Esta informação condiz com os dados encontrados e 
descritos em pesquisa anterior, que a maioria dos pacientes acometidos de ISC eram portadores de alguma doença crônica. Quando aplicada a correlação de Pearson, esta variável apresentouse com uma correlação fraca, ou seja, ela exerce relação com desencadeamento de ISC quando associada a outros fatores.

Dos pacientes que possuíam doenças crônicas dentro do grupo atingido, $50 \%$ eram pacientes hipertensos. Quando analisado de forma proporcional, visando à amostra total entre pacientes portadores de doenças crônicas e os não portadores de doenças crônicas, observouse que pacientes saudáveis representaram apenas $2,7 \%$ dos que desenvolveram ISC enquanto pacientes que possuíam doenças crônicas apareceram com 17,6\%.

A hipertensão, além de poder levar estes pacientes a outras morbidades, também retarda o processo de cicatrização, favorecendo o surgimento de ISC [14]. Desta forma, os dados relacionados a esta variável condizem com a pesquisa anterior, sugerindo que este seja um fator que caminha concomitante com o surgimento de ISC. Estaticamente analisada, a hipertensão arterial referiu uma correlação muito fraca com o desencadeamento de ISC, desta forma, não sendo um fator determinante para a mesma.

Durante a pesquisa, também foram avaliadas medidas realizadas no período préoperatório como o banho e a tricotomia. Todos os pacientes pertencentes ao grupo acometidos alegaram ter realizado banho pré-operatório.

Estudos indicam que o banho pré-operatório pode ser uma intervenção eficiente no combate às infecções do sítio cirúrgico, porém é indicado que ocorram dois banhos sequenciais utilizando clorexidina 4\%, para que tal medida siga eficiente [15]. Esta intervenção não foi seguida à risca pelos pacientes que compuseram a amostra, pois estes realizaram banho de aspersão comum. Diante destas informações, os pacientes não se tornaram menos suscetíveis a ISC.

Todos os pacientes com ISC relataram ter realizado tricotomia no local do procedimento, as tricotomias relatadas pelos pacientes foram realizadas em domicílio. A tricotomia é considerada um fator de risco para o surgimento da mesma por ocasionar possíveis escoriações, deixando uma porta de entrada para infecção na ferida cirúrgica, sendo indicada que haja inspeção da pele para identificar presenças de sinais flogísticos ocasionados no momento da tricotomia [16].

Diante destes dados sugere-se que, ao realizar a medida de tricotomia, os pacientes colaborem de forma inconsequente com o surgimento de ISC por serem leigos em relação a tal medida.

Em relação ao potencial de contaminação, dentre os pacientes afetados, $75 \%$ realizaram procedimentos potencialmente contaminados. Quanto mais limpos os procedimentos cirúrgicos, menos as chances de ocorrerem complicações como as infecções de sítio cirúrgico [17].

Os resultados apontam que esta variável está em conformidade com a referência anteriormente citada, já que dentre os pacientes acometidos, a maioria realizou procedimentos classificados como potencialmente contaminados, sendo sugerível que procedimentos como estes sejam mais suscetíveis a complicações como a ISC. Destes, $38 \%$ dos casos de ISC foram advindos do procedimento de histerectomia, classificado como um procedimento potencialmente contaminado.

A realização de histerectomias é frequente, uma vez que este é o procedimento ginecológico mais realizado em todo o mundo, mesmo com os avanços tecnológicos e o surgimento de técnicas menos invasivas e que ofertem menos riscos as pacientes, ainda é extremante utilizada [18]. Deste modo, os resultados obtidos mostram que a histerectomia é um procedimento muito realizado, uma vez que a maior parte da amostra realizou este procedimento, o que pode se atribuir ao fato do mesmo ter sido o mais atingido pela ISC.

Dentre este grupo de pacientes que desenvolveram ISC, $25 \%$ fizeram uso de dreno. $O$ uso de dreno representa um fator de risco, sendo caracterizado como uma porta de entrada de microrganismos [19]. Portanto, esta variável não teve tal relevância como vista em pesquisa anterior, neste estudo, a razão para este dado não ter sido tão relevante pode estar relacionada a pouca quantidade da amostra que fizeram o uso de dreno.

Outro fator avaliado foi uso de SVD, exatos $25 \%$ dos pacientes que compõe este grupo também fizeram o uso deste dispositivo para eliminação vesical. De acordo com publicação anterior, este procedimento caracteriza-se como invasivo, sendo desta forma um fator de risco para complicações cirúrgicas, dentre elas a ISC [20].

Variável esta que não obteve grandes percentuais dentro do grupo de pacientes afetados, assim como o dreno. Este fato pode ser atribuído a pouca quantidade de pacientes que fizeram o uso de SVD. 
Dentre os pacientes com ISC, também foram avaliados de acordo com a classificação de ASA, considerado um fator de risco, e $75 \%$ foram classificados como ASA II, representando a maioria da amostra dos pacientes atingidos. Estudo antecedente afirma que o desenvolvimento de IRAS ocorre com maior frequência em pacientes mais debilitados, sendo considerada como um fator de risco para as mesmas [21].

Diante destas informações, os resultados relatados seguem os subsídios encontrados dentro da referência anterior, logo pacientes classificados como ASA II representaram a maioria dos pacientes afetados.

Alusivo ao uso de profilaxia antimicrobiana, todos os pacientes que desenvolveram infecção na ferida cirúrgica fizeram uso de antibiótico durante o pós-operatório. Estudo anterior cita que, para uma defesa eficaz, a profilaxia antimicrobiana deve ocorrer dentro do préoperatório, exceto em cirurgias limpas e no período pós-operatório nas primeiras 24 horas, sendo uma medida eficaz que visa prevenir o desenvolvimento de ISC [22].

Levando esta informação em consideração, os pacientes não cumpriram as recomendações profiláticas antimicrobianas adequadas, tornando-se mais aptos para adquirirem ISC, já que não havia relatos de administração profiláticas no pré-operatório. É importante ressaltar que além destes fatores já citados, existem outros fatores tanto extrínsecos como intrínsecos que podem comprometer a eficácia das medidas profiláticas e terapêuticas.

Sobre o retorno hospitalar, dentre os indivíduos atingidos, a maioria $(88 \%)$ relatou ter retornado à unidade de saúde devido a algum evento relacionado à ferida cirúrgica. Em estudos anteriores, a evidência de diagnóstico de ISC está relacionada à necessidade de retorno [23]. Desta forma, os resultados relatados coincidem com o estudo anterior, uma vez que a maior porcentagem dos pacientes que desenvolveram ISC retornaram à unidade referindo algum evento adverso na ferida cirúrgica, propondo que o fato de retorno hospitalar pode estar associado àqueles pacientes que desenvolveram ISC.

Ao aplicar esta variável na correlação, observa-se que a mesma apresenta uma correlação muito fraca, não sendo um fator determinante para o desenvolvimento de ISC, porém na presença de outros fatores pode possuir certa influência.

A incidência de infecção resultante da pesquisa foi de $7,5 \%$, utilizando o cálculo de incidência da ANVISA, sendo um percentual alto em comparação com o resultado encontrado na pesquisa utilizada como base, elaborada por Aguiar et al. [3], intitulada como "Fatores associados à infecção de sítio cirúrgico em um hospital na Amazônia ocidental brasileira", sendo que nesta a prevalência foi de $3,68 \%$.

Diante destes resultados, algumas características presentes na amostra apresentaram variáveis com maior incidência dentro do grupo que desenvolveu ISC, dentre estas variáveis encontra-se sexo feminino, baixa escolaridade, portadores de doenças crônicas com destaque para HAS, e pacientes classificados como ASA II.

A execução das medidas realizadas no período pré-operatório como a tricotomia e o banho de forma equivocada foram fatores presentes nos pacientes que desenvolveram ISC. Dentre outros elementos analisados relacionados diretamente aos procedimentos realizados, as histerectomias e os procedimentos potencialmente contaminados também se destacaram como fatores de incidência nos pacientes que desenvolveram ISC.

Com os resultados levantados foi possível identificar fatores que podem estar associados e que podem contribuir com o desenvolvimento de ISC, sendo estes tanto de vertentes intrínsecas como extrínsecas. Deste modo, os profissionais da área da saúde precisam ser informados sobre estes fatores de risco para estarem alerta, sendo possível a formulação de cuidados e orientações que possam contribuir com a diminuição da incidência de ISC.

O resultado da pesquisa também contribui com os bancos de dados, sendo útil para profissionais da saúde, acadêmicos e para a população que tem acesso a informação. Contribui com estudos anteriores e abre portas para próximos estudos relacionados a esta temática, correspondendo como material de embasamento teórico para futuras pesquisas.

Durante o trajeto da pesquisa houve condições que limitaram sua realização, como a perda de contato de pacientes no momento do contato telefônico, pacientes em zona rurais com o difícil acesso a rede telefônica, cancelamento de cirurgias eletivas, pacientes que não compareceram para a realização dos procedimentos. Entretanto os pacientes participantes foram satisfatórios para efetivação do estudo. 
1. Nogueira Junior $\mathrm{C}$. Sistemas de informação relacionada a assistência à saúde IRAS: Delineando a realidade das regiões sudeste e sul do Brasil. São Paulo: Universidade de São Paulo; 2013. [citado 2017 set 07]. Disponível em: https://www.teses.usp.br

2. Barros CSM. Custos atribuídos às infecções de sítio cirúrgico em um hospital universitário em Salvador-Bahia, 2016. Salvador: Fundação Oswaldo Cruz; 2016. CDU 616-089(813.8). [citado 2017 Ago 10]. Disponível em: http://www.arca.fiocruz.br

3. Aguiar APL, Prado PR, Opitz SP, Optiz SP, Faro ARMC. Fatores associados à infecção de sítio cirúrgico em um hospital na Amazônia ocidental brasileira. Rev SOBECC, São Paulo, SP, Brasil, 2012. [citado 2017 set 11]. Disponível em: http://www.revista.sobecc.org.br

4. Martins T, Amante LN, Virtuoso JF, Girondi JBR, Nascimento ERP, Nascimento KC. Pré-operatório de cirurgias potencialmente contaminadas: Fatores de risco para infecção do sítio cirúrgico. Acta Paul Enferm 2017;30(1):16-24. https://doi.org/10.1590/1982-0194201700004

5. Ercole FF, Chianca TCM. Infecção de sítio cirúrgico em pacientes submetidos a artroplastias de quadril. Rev Latinoam Enferm 2002;10(2):157-65. https://doi.org/10.1590/S0104-11692002000200006

6. Agência Saúde. Ministério da Saúde Portal da Saúde 2015. [citado 2017 set 5]. https://www.saude.gov.br

7. Portal da Saúde - Ministério da Saúde 2017. [citado 2017 set 9]. https://www.saude.gov.br

8. ANVISA. Agência Nacional de Vigilância Sanitária. Critérios Nacionais de Infecções relacionadas à assistência à saúde. Março de 2009. [citado 2017 set 15]. https://www.anvisa.gov.br

9. Oliveira MM, Daher DV, Silva JLL, Andrade SSCA. A saúde do homem em questão: busca por atendimento na atenção básica de saúde. Ciênc Saúde Coletiva 2015;20(1):273-8. https://doi.org/10.1590/1413-81232014201.21732013

10. Bellusse GC, Ribeiro JC, Campos FR, Poveda VB, Galvão CM. Fatores de risco de infecção da ferida operatória em neurocirurgia. Acta Paul Enferm 2017;28(1). https://doi.org/10.1590/1982-0194201500012

11. Feitosa RGF, Fernandes FAM, Narciso Júnior J, Araújo Júnior ON, Costa FA, Cavalcante LDW. Análise da incidência de infecção de sítio cirúrgico em cirurgias oncológicas do aparelho digestivo no Hospital Geral de Fortaleza. Medicina (Ribeirão Preto) 2014;47(2):157-64. https://doi.org/10.11606/issn.2176-7262.v47i2p157-164

12. Cavichio BV, Pompeo DA, Oller GASAO, Ross LA. Tempo de cessação do tabagismo para a prevenção de complicações na cicatrização de feridas cirúrgicas. Rev Esc Enferm. USP 2014;48(1):174-80. https://doi.org/10.1590/S0080-623420140000100022

13. Borges ES. Instrumento para controle e prevenção de infecção de sítio cirúrgico em neurocirurgia [Dissertação]. Niterói: Escola de Enfermagem Aurora de Afonso Costa; 2016. 92 f. [citado 2017 set 20]. Disponível em: https://app.uff.br/riuff/handle/1/3063

14. Santos PVF, Jesus KBD, Santana KISPD, Nogueira EC, Cariri LS, Brito FPG. Infecção do sítio cirúrgico em pacientes no pós-operatório de cirurgias ortopédicas eletivas. Aracaju: Interfaces Científicas - Saúde e Ambiente 2017;5(2).

15. Franco LMC, Almeida AGIA, Duarte GMH, Lamounier L, Pinto TS, Pereira PFS, et al. Efeitos do banho pré-operatório na prevenção de infecção cirúrgica: Estudo clínico piloto. Rev Min Enferm 2017;2(1);1053. https://doi.org/10.5935/1415-2762.20170063

16. Rodrigues $\mathrm{E}$. Assistência de enfermagem no pré e pós-operatório mediato ao utente em risco de desenvolver uma infecção do local cirúrgico. Cabo Verde: Universidade do Mindelo Escola Superior de Saúde 2015. [citado 2017 ago 20]. Disponível em: http://hdl.handle.net/10961/4678

17. Winck DL, Cosentino SF, Prestes M, Andrade A, Nogueira GM. Índice de prevalência de infecção em feridas operatórias ortopédicas e traumatológicas. Revista Espaço Ciência \& Saúde 2015;3:46-58. [citado 2017 set 28]. Disponível em: https://www.semanticscholar.org

18. Brito LGO, Silva JCR, Nogueira AA. Reflexões sobre o impacto causado pelo alerta do Food and Drug Administration (FDA) americano sobre morcelamento eletromecânico uterino e/ou de miomas. Rev Bras Ginecol Obstet 2015;37(7):299-301. https://doi.org/10.1590/S0100-720320150005428 
19. Carvalho TA, Campos MPA, Lobo IMF. Riscos e complicações associadas à infecção do sítio cirúrgico: Um estudo de coorte. Aracaju: Universidade Federal de Sergipe UFS 2016. [citado 2017 ago 27]. http://ri.ufs.br/ispui/handle/riufs/4985

20. Fusco SFB, Massarico NM, Alves MVMFF, Fortaleza CMCB, Pavan ECP, Palhares VC, et al. Infecção de sítio cirúrgico e seus fatores de risco em cirurgias de cólon. Rev Esc Enferm USP 2016;50(1):43-9. [citado 2017 set 28]. https://doi.org/10.1590/S0080623420160000100006

21. Carvalho RLR. Fatores de risco para infecção de sítio cirúrgico em procedimentos gerais em um hospital público de Belo Horizonte, Minas Gerais - Um estudo de incidência. Belo horizonte: Universidade Federal de Minas Gerais Escola de Enfermagem 2014. [citado 2017 set 24]. Disponível em: http://www.enf.ufmg.br

22. Gebrim CFL, Rodrigues JG, Queiroz MNR, Barreto RASS, Palos MAP. Análise da profilaxia antimicrobiana para a prevenção da infecção do sítio cirúrgico em um hospital Do centro-oeste brasileiro. Ciência y Enfermeria 2014;(2):103-15. [citado 2017 Ago 28]. https://scielo.conicyt.cl

23. Ribeiro JC, Santos CB, Bellusse GC, Rezende VF, Galvão CM. Ocorrência e fatores de risco para infecção de sítio cirúrgico em cirurgias ortopédicas. Acta Paul Enferm 2013;26(4);353-9. https://doi.org/10.1590/S0103-21002013000400009 